\title{
1 A local model for the limiting configuration of 2 interfacial solitary waves
}

\author{
3 X. Guan ${ }^{1,2}$, J.-M. Vanden-Broeck ${ }^{2}$, Z. Wang ${ }^{1} \dagger$ and F. Dias $^{3}$ \\ $4{ }^{1}$ Institute of Mechanics, Chinese Academy of Sciences, Beijing 100190, China \\ $5 \quad{ }^{2}$ Department of Mathematics, University College London, London WC1E 6BT, UK \\ $6{ }^{3}$ School of Mathematics and Statistics, University College Dublin, Belfield, Dublin, Ireland \\ 7 (Received xx; revised xx; accepted xx)
}

8 The limiting configuration of interfacial solitary waves between two homogeneous fluids 9 consisting of a sharp $120^{\circ}$ angle with an enclosed bubble of stagnant heavier fluid on top 10 is investigated numerically. We use a boundary integral equation method to compute the 11 almost limiting profiles which are nearly self-intersecting and thus extend the work of Pullin 12 \& Grimshaw (Phys. Fluids 31, 1988, 3550-3559) by obtaining the overhanging solutions for 13 very small density ratios. To further study the local configuration of the limiting profile, we propose a reduced model that replaces the $120^{\circ}$ angle with two straight solid walls intersecting at the bottom of the bubble. Using a series truncation method, a one-parameter family of solutions depending on the angle between the two solid walls (denoted by $\gamma$ ) is found. When $\gamma=2 \pi / 3$, it is shown that the simplified model agrees well with the near-limiting wave profile if the density ratio is small, and thus provides a good local approximation to the assumed limiting configuration. Interesting solutions for other values of $\gamma$ are also explored.

\section{Introduction}

It was conjectured by Stokes that for two-dimensional deep surface gravity waves, there exists a family of periodic travelling waves that terminates at an 'extreme wave' as it reaches the maximum amplitude. Such limiting configuration, termed the Stokes highest wave, can be characterised by a stagnation point at the crest and an enclosed angle of $120^{\circ}$. The existence of the Stokes highest wave was extensively studied by a variety of authors from asymptotic and numerical perspectives (Havelock 1918; Yamada 1957a; Longuet-Higgins 1973; Schwartz 1974; Vanden-Broeck \& Schwartz 1979), and ultimately proved rigorously by Amick et al. (1982). It was also pointed out by Amick et al. (1982) that the Stokes conjecture holds regardless of wavelength and water depth, and in particular, in the limit of infinite wavelength, the extreme solitary wave on water of finite depth features the same limiting crest angle. Yamada (1957b) is the first known author to have solved for the limiting solitary wave numerically (see the book by Okamoto \& Shōji (2001) for a detailed description of Yamada's method). Lenau (1966) used a series truncation method to compute the same wave. Hunter \& Vanden-Broeck (1983) improved Lenau's results.

For waves between two homogeneous fluids, the sharp crest of $120^{\circ}$ cannot serve as the limiting configuration of the interface since it would result in an infinite velocity in the upper fluid (Meiron \& Saffman 1983). Attempts to understand the limiting profile of interfacial periodic waves were made by Saffman \& Yuen (1982), Meiron \& Saffman (1983) and Turner \& Vanden-Broeck (1986), who numerically discovered the overhanging structure (i.e. multivalued wave profiles). Meiron \& Saffman (1983) further asserted that the related

$\dagger$ Email address for correspondence: zwang@imech.ac.cn 


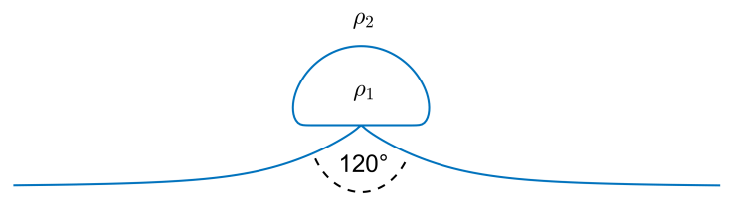

$\rho_{1}$

Figure 1: A possible limiting configuration for overhanging interfacial solitary waves: a sharp $120^{\circ}$ angle with a closed fluid bubble on top of it.

limiting profile would become self-intersecting. Grimshaw \& Pullin (1986) obtained the (almost) self-intersecting solutions when the upper fluid is of infinite depth. They conjectured that a possible extreme profile features a stagnant fluid bubble on top of a $120^{\circ}$ angle. Recently, Maklakov \& Sharipov (2018) conducted a thorough numerical study on the almost limiting configuration between semi-infinite fluid layers. They obtained highly accurate solutions, providing reliable evidence for the extreme profile predicted by Grimshaw \& Pullin (1986). Maklakov (2020) discussed the transition from interfacial waves to surface waves when the density ratio tends to zero. For interfacial solitary waves, Amick \& Turner (1986) proved that a possible extreme configuration is an internal front developed from flattening and unlimited broadening of the solitary pulse as the wave speed approaches a limiting value. This theoretical result was verified later by several numerical computations (see, e.g., Funakoshi \& Oikawa 1986; Turner \& Vanden-Broeck 1988; Rusås \& Grue 2002). However, Amick \& Turner (1986) also showed that the interface could develop a vertical tangent indicating the existence of multi-valued solutions, thus provided another possibility. Pullin \& Grimshaw (1988) computed the interfacial solitary waves with an overhanging structure and suggested the existence of a self-intersecting profile. However, they could not obtain overhanging waves when the density ratio is smaller than 0.0256 , which was explained by a rapid shrinking of the overhanging structure when the density ratio is small and is further decreased, and therefore more grid points are required to capture it.

In the current paper, we consider interfacial solitary waves between two fluids of finite depths. A boundary integral equation method is used to calculate overhanging solutions and the results of Pullin \& Grimshaw (1988) are extended to very small density ratios. Based on numerical results and local analysis, we suggest a possible limiting configuration featuring a $120^{\circ}$ angle-bubble structure, akin to the periodic case (see figure 1). A reduced model, which replaces the curved angle with two straight rigid walls intersecting at the bottom of the fluid bubble, is proposed and numerically solved using a series truncation method. It turns out that the simplified model provides a good local approximation for the cases of a small density ratio when the upper layer is deep enough. The reduced model can also be applied to periodic interfacial waves due to its local nature.

\section{Mathematical formulation}

We consider a two-dimensional solitary wave travelling at speed $c$ between two incompressible and inviscid fluids, bounded above and below by horizontal solid walls. We take a frame of reference moving with the wave. The $x$-axis is parallel to the rigid walls. The level $y=0$ is chosen as the undisturbed level of the interface and gravity is assumed to act in the negative $y$-direction. We denote by $h_{i}$ and $\rho_{i}(i=1,2)$ the depth and density in each fluid layer, where subscripts 1 and 2 refer to fluid properties associated with the lower and upper fluid layers, respectively. Velocities are measured in units of $c$ and lengths in units of $h_{1}$. The motion 
of each fluid is assumed to be irrotational, thus we introduce velocity potentials $\phi_{1}$ and $\phi_{2}$, which satisfy the Laplace equation in the corresponding fluid layers

$$
\phi_{i, x x}+\phi_{i, y y}=0, \quad i=1,2 .
$$

At the interface, the kinematic and dynamic boundary conditions can be expressed as

$$
\phi_{i, y}-\phi_{i, x} \eta_{x}=0, \quad i=1,2,
$$

$$
R\left|\nabla \phi_{2}\right|^{2}-\left|\nabla \phi_{1}\right|^{2}+\frac{2(R-1)}{F^{2}} \eta=R-1,
$$

where $R=\rho_{2} / \rho_{1}<1$ for a density-stable configuration, $F=c / \sqrt{g h_{1}}$ is the Froude number, and $g$ is the acceleration due to gravity. The boundary conditions at the solid walls read

$$
\begin{aligned}
& \phi_{1, y}=0, \quad \text { at } \quad y=-1, \\
& \phi_{2, y}=0, \quad \text { at } \quad y=h,
\end{aligned}
$$

where $h=h_{2} / h_{1}$ stands for the dimensionless depth of the upper layer. To describe a solitary wave in the comoving frame we require $\eta \rightarrow 0$ and $\phi_{i, x} \rightarrow-1$ as $|x| \rightarrow \infty$ and, additionally, we confine our attention to symmetric waves with the crest at $x=0$.

\section{Numerical results via a boundary integral method}

Following Sha \& Vanden-Broeck (1993), we reformulate the problem by using the Cauchy integral formula

$$
\zeta\left(z_{0}\right)+1=\frac{1}{\mathrm{i} \pi} \oint_{C} \frac{\zeta(z)+1}{z-z_{0}} \mathrm{~d} z
$$

where $z=x+\mathrm{i} y$ is the complex coordinate, $\zeta=\phi_{x}-\mathrm{i} \phi_{y}=u-\mathrm{i} v$ is the complex velocity, and $C$ stands for the boundary of the considered domain. We parameterise the interface by the arc length $s \in(-\infty, \infty)$ and let $s=0$ at $x=0$. By applying the Cauchy integral formula to the lower and upper fluid layers respectively and taking the real parts, one obtains

$$
\begin{aligned}
& \pi\left[u_{1}(\sigma)+1\right] \\
& =\int_{0}^{\infty} \frac{\left[\left(u_{1}(s)+1\right) x^{\prime}(s)+v_{1}(s) \eta^{\prime}(s)\right][2+\eta(s)+\eta(\sigma)]-\eta^{\prime}(s)[x(s)-x(\sigma)]}{[x(s)-x(\sigma)]^{2}+[2+\eta(s)+\eta(\sigma)]^{2}} \mathrm{~d} s \\
& +\int_{0}^{\infty} \frac{\left[\left(u_{1}(s)+1\right) x^{\prime}(s)+v_{1}(s) \eta^{\prime}(s)\right][2+\eta(s)+\eta(\sigma)]-\eta^{\prime}(s)[x(s)+x(\sigma)]}{[x(s)+x(\sigma)]^{2}+[2+\eta(s)+\eta(\sigma)]^{2}} \mathrm{~d} s \\
& +\int_{0}^{\infty} \frac{\left[\left(u_{1}(s)+1\right) x^{\prime}(s)+v_{1}(s) \eta^{\prime}(s)\right][\eta(s)-\eta(\sigma)]-\eta^{\prime}(s)[x(s)-x(\sigma)]}{[x(s)-x(\sigma)]^{2}+[\eta(s)-\eta(\sigma)]^{2}} \mathrm{~d} s \\
& +\int_{0}^{\infty} \frac{\left[\left(u_{1}(s)+1\right) x^{\prime}(s)+v_{1}(s) \eta^{\prime}(s)\right][\eta(s)-\eta(\sigma)]-\eta^{\prime}(s)[x(s)+x(\sigma)]}{[x(s)+x(\sigma)]^{2}+[\eta(s)-\eta(\sigma)]^{2}} \mathrm{~d} s,
\end{aligned}
$$

$$
\begin{aligned}
& \pi\left[u_{2}(\sigma)+1\right] \\
& =\int_{0}^{\infty} \frac{\left.\left[\left(u_{2}(s)+1\right) x^{\prime}(s)+v_{2}(s) \eta^{\prime}(s)\right][2 h-\eta(s)-\eta(\sigma)]+\eta^{\prime}(s)[x(s)-x(\sigma))\right]}{[x(s)-x(\sigma)]^{2}+[2 h-\eta(s)-\eta(\sigma)]^{2}} \mathrm{~d} s \\
& +\int_{0}^{\infty} \frac{\left.\left[\left(u_{2}(s)+1\right) x^{\prime}(s)+v_{2}(s) \eta^{\prime}(s)\right][2 h-\eta(s)-\eta(\sigma)]+\eta^{\prime}(s)[x(s)+x(\sigma))\right]}{[x(s)+x(\sigma)]^{2}+[2 h-\eta(s)-\eta(\sigma)]^{2}} \mathrm{~d} s \\
& -\int_{0}^{\infty} \frac{\left[\left(u_{2}(s)+1\right) x^{\prime}(s)+v_{2}(s) \eta^{\prime}(s)\right][\eta(s)-\eta(\sigma)]-\eta^{\prime}(s)[x(s)-x(\sigma)]}{[x(s)-x(\sigma)]^{2}+[\eta(s)-\eta(\sigma)]^{2}} \mathrm{~d} s
\end{aligned}
$$




$$
-\int_{0}^{\infty} \frac{\left[\left(u_{2}(s)+1\right) x^{\prime}(s)+v_{2}(s) \eta^{\prime}(s)\right][\eta(s)-\eta(\sigma)]-\eta^{\prime}(s)[x(s)+x(\sigma)]}{[x(s)+x(\sigma)]^{2}+[\eta(s)-\eta(\sigma)]^{2}} \mathrm{~d} s,
$$

where the Schwarz reflection principle and the symmetry of the interface with respect to the $y$-axis are used. For the computations, equations (3.2) and (3.3) are calculated over a finite interval $[0, L]$ with $L$ large. Two sets of mesh grids

$$
\begin{aligned}
s_{i} & =\frac{(i-1) L}{N-1}, \quad i=1,2, \ldots, N, \\
\sigma_{i} & =\frac{s_{i}+s_{i+1}}{2}, \quad i=1,2, \ldots, N-1,
\end{aligned}
$$

are introduced. Then $2 N-2$ algebraic equations can be obtained via evaluating the integrals at $\sigma_{i}$ by the trapezoid rule. The boundary conditions at the interface, (2.2) and (2.3), as well as the arc length equation

$$
x^{\prime 2}(s)+\eta^{\prime 2}(s)=1
$$

are evaluated at $s_{i}$, resulting in $4 N$ algebraic equations. Since there are $6 N+1$ unknowns, namely $x^{\prime}\left(s_{i}\right), \eta^{\prime}\left(s_{i}\right), u_{1}\left(s_{i}\right), v_{1}\left(s_{i}\right), u_{2}\left(s_{i}\right), v_{2}\left(s_{i}\right)$ and $F$ (for a given wave height $H$ ), three additional equations are needed to close the system:

$$
u_{1}(L)=-1, \quad \eta^{\prime}(0)=0, \quad \text { and } \quad \eta(0)=H .
$$

The unknowns at $\sigma_{i}$ can be obtained by means of a four-point interpolation formula. For fixed values of $R$ and $h$, we calculate solitary waves via Newton's method with an initial guess being a small-amplitude Gaussian profile. The iteration process is repeated until the maximum residual error is less than $10^{-8}$. We slowly change the value of $H$ (or $F$ ) and use the known solutions as the initial guess, thus solution branches can be systematically explored.

Numerical results indicate that unlimited broadening of the central core of solitary waves that ultimately turn into conjugate flows is likely to occur for small $h$ (see Turner \& Vanden-Broeck 1988). In order to obtain overhanging solutions, we choose large values for $h$ ( $h=80$ say) in the subsequent computations. Three speed-amplitude bifurcation curves are shown in Figure 2(a) for the density ratios $R=0.1,0.2,0.3$. Accordingly, the numerical calculations are performed with $L=40,50,100$ and $N=1200,800$, 500. Some typical wave profiles are plotted in Figure 2(b,c,d). In general, it is found that along the bifurcation curve solitary waves gradually steepen, reach the maximum speed corresponding to the first turning point, and form a mushroom-shaped solitary pulse ultimately. It is observed that multiple turning points may exist on the same branch where the overhanging structure oscillates between closing and opening before it reaches the limiting configuration. The wave profile in the bottom figure of $2(\mathrm{c})$ is the closest to the proposed limiting configuration shown in Figure 1 among all the numerical solutions that we obtained. Our numerical results agree well with those found by Pullin \& Grimshaw (1988) who conjectured that all solitary waves for small density ratios would develop an overhanging structure. Solitary waves with an overhanging structure can also be found for other values of $R$, and for instance, Figure 3 shows the numerical results obtained based on two sets of parameters: $(R, L, N)=(0.01,8,2000)$ and $(0.6,200,290)$. It is noted that solutions for $R=0.01$ extend the result of Pullin \& Grimshaw (1988) since they could not get overhanging profiles for $R<0.0256$ due to numerical difficulties.

Based on the aforementioned numerical evidence, it is reasonable to conjecture that the limiting configuration is a self-intersecting interface consisting of a sharp angle and a closed fluid bubble as shown in Figure 1. To verify this assertion, we plot the velocity magnitude distributions (i.e. $u_{1,2}^{2}+v_{1,2}^{2}$ ) at the interface in Figure 4(a) for $R=0.15$ and $h=80$. It is clear that there are two segments where velocities above or below the interface are almost 

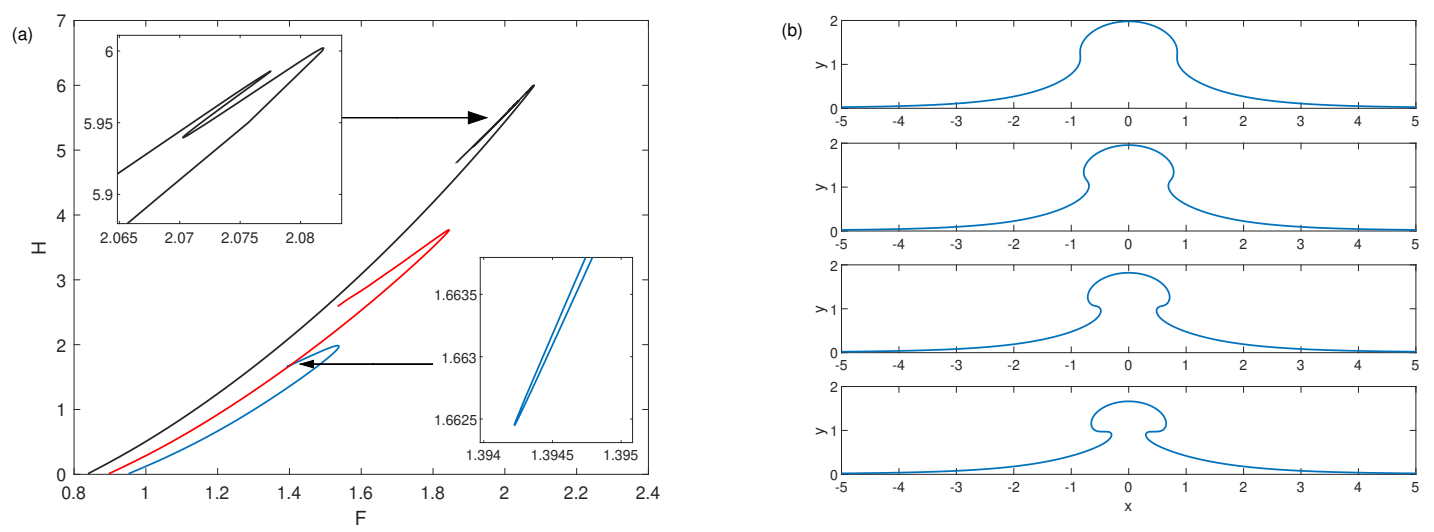

(c)

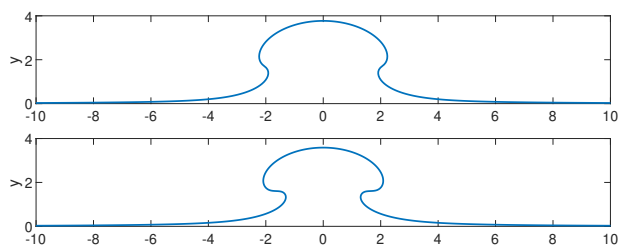

(d)
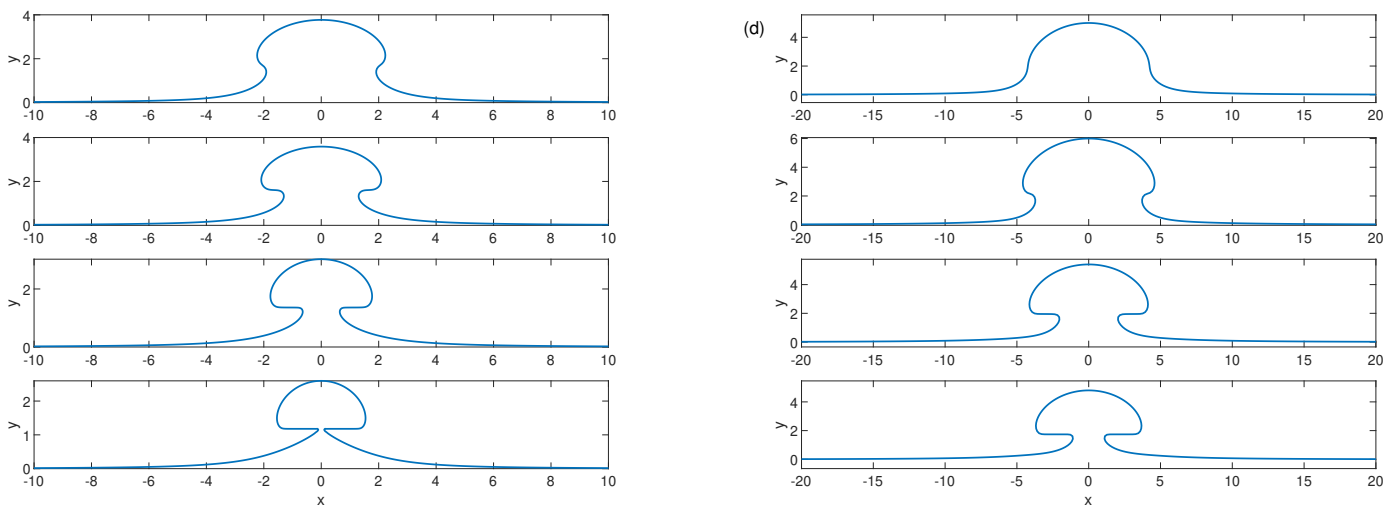

Figure 2: (a) Speed-amplitude bifurcation curves for $h=80$ and $R=0.1$ (blue), $R=0.2$ (red), $R=0.3$ (dark). (b-d) Typical overhanging profiles for $R=0.1,0.2,0.3$ respectively.
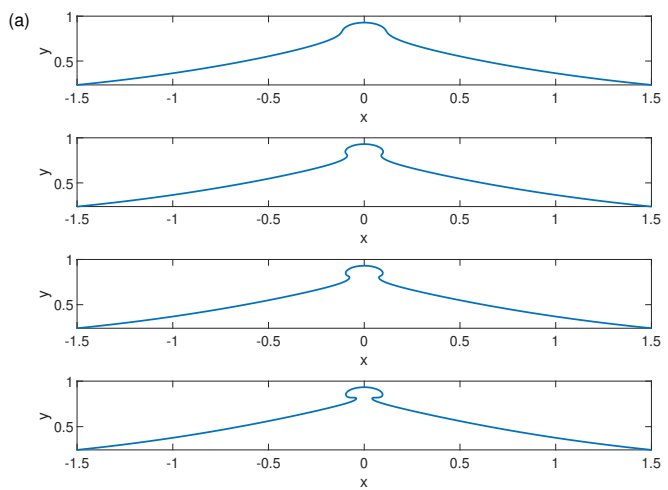

(b)
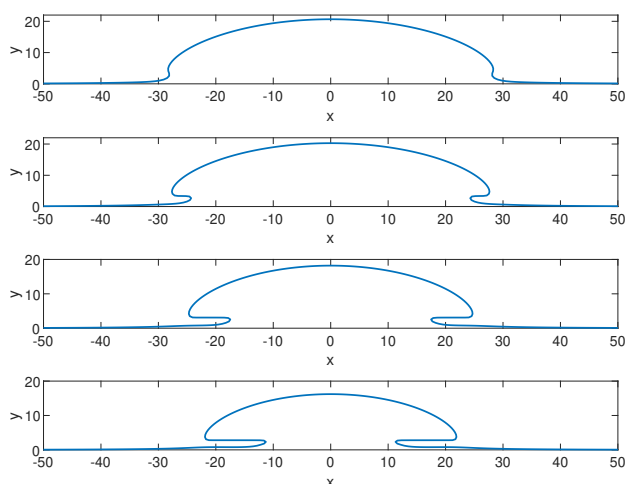

Figure 3: Overhanging waves for $h=80$ and (a) $R=0.01$, (b) $R=0.6$.

zero. The common segment on which $u_{1,2}^{2}+v_{1,2}^{2}<0.005$ is labeled by a thick black line in (a) and correspondingly highlighted on the wave profile in (b). Consequently, for the limiting configuration shown in Figure 1, if it exists, the fluid inside the bubble should be stationary since closed streamlines are not allowed for irrotational flows. Based on a similar argument of the Stokes highest wave, the sharp corner attached to the fluid bubble should be of an interior angle of $120^{\circ}$ with the vertex being a stagnation point. On the other hand, Bernoulli's equation at the stagnation point implies $y_{0}=F^{2} / 2$ for all density ratios, where $y_{0}$ 

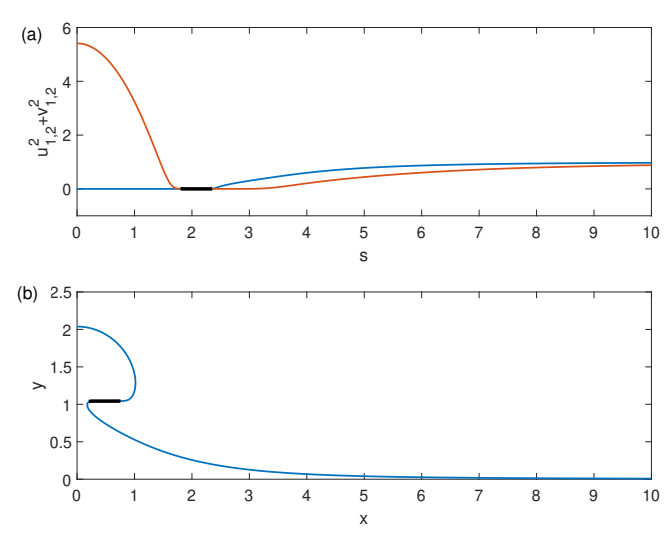

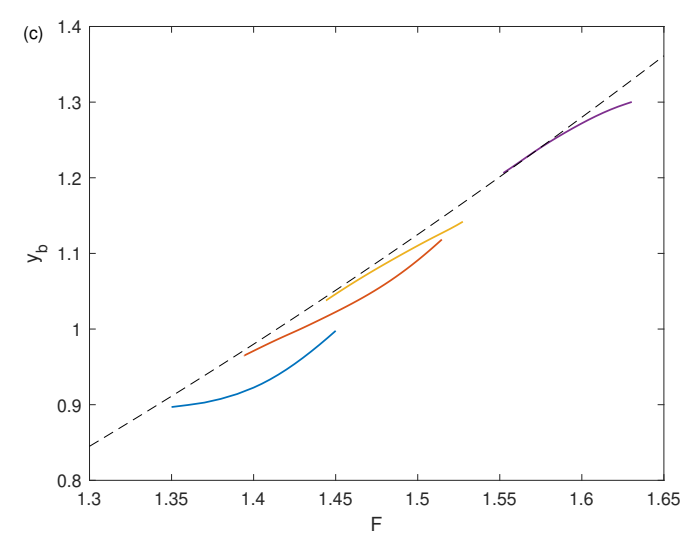

Figure 4: (a) Interfacial velocity magnitude of the upper fluid (red) and lower fluid (blue) for $R=0.15$ and $h=80$. The segment on which $u_{1,2}^{2}+v_{1,2}^{2}<0.005$ is labeled by the black thick line. (b) Wave profile associated with (a), and the black part of the interface corresponds to $u_{1,2}^{2}+v_{1,2}^{2}<0.005$. (c) Numerical relations between $y_{b}$ and $F$ for $R=0.08$ (blue), $R=0.1$ (red), $R=0.15$ (yellow), and $R=0.2$ (purple), together with the theoretical prediction $y_{0}=F^{2} / 2$ (dashed line). $y_{b}$ denotes the vertical coordinate of the bubble bottom, and $y_{0}$ is the theoretical vertical coordinate of the stagnation point.

is the vertical coordinate of the vertex. The theoretical prediction $y_{0}=F^{2} / 2$ is plotted as the dashed line in Figure 4(c). Typical numerical values for $y_{b}(F)$ are shown in the same figure as solid lines, where $y_{b}$ is the vertical coordinate of the flat bottom of the fluid bubble, namely the part labeled as black in Figure 4(b). The four curves correspond to $R=0.08,0.1,0.15,0.2$.

\section{A simplified model}

Although the almost self-intersecting solutions can be obtained by the boundary integral equation method, the appearance of the singularity, i.e. the $120^{\circ}$ angle, is a formidable difficulty to overcome. As one can see from Figures 2 and 3, the overhanging structure is fully localised and shrinks rapidly when the value of $R$ is decreased and, furthermore, the local structure beneath the bubble looks very much like an obtuse angle between two straight lines if the density ratio is small, e.g. $R=0.01$. Motivated by these observations, we attempt to propose a simplified model to describe the local structure of the limiting configuration for small density ratios.

As shown in the simplified model of Figure 5, the end points A and C, which respectively represent upstream and downstream sides of a flow, are assumed to extend to infinity. The lines $\mathrm{OA}$ and $\mathrm{OC}$ are supposed to be solid walls where impermeability boundary conditions need to be satisfied. The angle $\gamma$ is considered to be a parameter, and $\gamma=2 \pi / 3$ is the relevant one to model interfacial waves. This is because the flow inside the angle $\mu$ approaches a stagnation flow as the point $\mathrm{O}$ is approached, where $\mu$ is the angle between the solid wall and the bubble bottom (see Figure 5). The flow of fluid 1 inside the angle $\gamma$ near the point $O$ reduces then to the local flow considered by Stokes to model surface waves. It then follows that $\gamma=2 \pi / 3$. We note that the bottom part of the bubble near O is horizontal, so that $\mu=(\pi-\gamma) / 2$. This can be justified by a local analysis of the flow inside the angle $\mu$, a flow bounded above by a free surface and below by a solid wall. It can be shown that the free surface has to be horizontal at $O$ (the only other possibility is the value $\mu=2 \pi / 3$ which is not relevant here), and the interested reader is referred to the third chapter of Vanden-Broeck (2010) for details. 


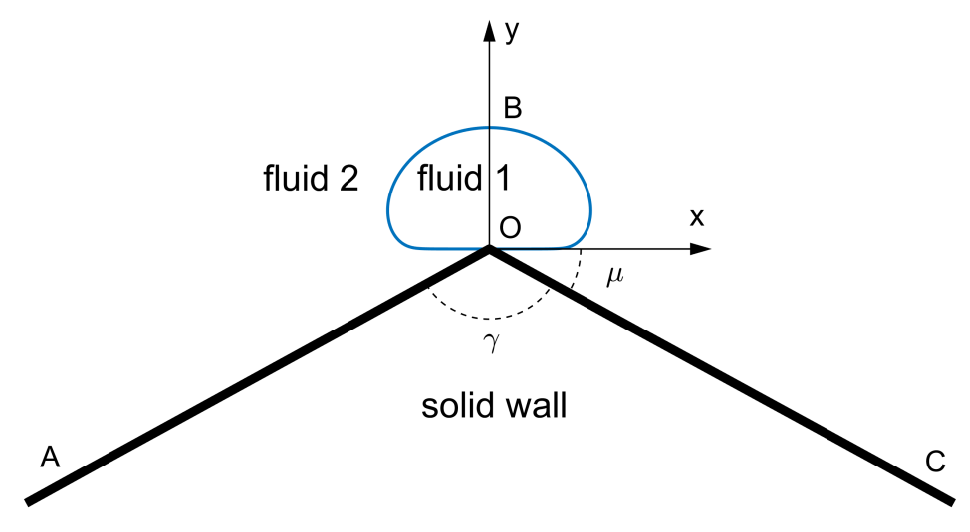

Figure 5: A simplified model: two straight solid walls intersect at the origin forming an angle $\gamma$ and a closed fluid bubble with flat bottom is on top of the angle.

For the sake of convenience, the origin of the Cartesian coordinate system is set to coincide with the angle vertex $\mathrm{O}$, with the $y$-axis pointing upward, and the summit of the bubble is label as B. Since the fluid inside the bubble is stationary, Bernoulli's equation now reads

$$
\frac{\rho_{2}}{2}\left(u_{2}^{2}+v_{2}^{2}\right)+\left(\rho_{2}-\rho_{1}\right) g \eta=0 .
$$

Our aim is to find the shape of the fluid bubble as well as the velocity potential $\phi_{2}$. This is a single layer problem since the fluid status beneath the interface is either known or irrelevant.

To solve the problem, we introduce the complex velocity potential $f=\phi_{2}+\mathrm{i} \psi$, with $\psi$ being the stream function. The value of $\psi$ at the interface and along the solid walls as well as $\phi_{2}(\mathrm{~B})$ are set to zero. It is noted the origin is actually the intersection of two walls, and hence we denote by $\mathrm{O}_{-}$and $\mathrm{O}_{+}$the left- and right-hand limits when approaching $\mathrm{O}$ along the corresponding walls and let $\Phi=\phi_{2}\left(\mathrm{O}_{+}\right)=-\phi_{2}\left(\mathrm{O}_{-}\right)$due to symmetry. We then non-dimensionalise the system by choosing $\left(\Phi^{2} / g\right)^{1 / 3}$ and $(\Phi g)^{1 / 3}$ as characteristic length and velocity scales, respectively. Following the work of Daboussy et al. (1998), we solve the problem by using the series truncation method. We introduce a transformation

$$
f=-\frac{1+t^{2}}{2 t}
$$

which maps the upper half $f$-plane (i.e. the domain occupied by the lighter fluid) onto the upper half unit disk in the complex $t$-plane. The images of $\mathrm{A}, \mathrm{O}_{-}, \mathrm{B}, \mathrm{O}_{+}, \mathrm{C}$ labelled in Figure 5 are $t=0,1, \mathrm{i},-1,0$. The complex velocity $\zeta=u_{2}-\mathrm{i} v_{2}$ is analytic everywhere except at $t=0$ and $t= \pm 1$, where the asymptotic behaviors are

$$
\begin{gathered}
\zeta \sim t^{1-\frac{\gamma}{\pi}}, \quad \text { as } t \rightarrow 0, \\
\zeta \sim\left(1-t^{2}\right)^{2-\frac{2 \mu}{\pi}}, \quad \text { as } t \rightarrow \pm 1,
\end{gathered}
$$

with $\mu=\frac{\pi-\gamma}{2}$. Therefore, the complex velocity $\zeta$ can be expressed as

$$
\zeta=\mathrm{e}^{\mathrm{i} \frac{\gamma-\pi}{2}} t^{1-\frac{\gamma}{\pi}}\left(1-t^{2}\right)^{2-\frac{2 \mu}{\pi}} \xi
$$

where $\xi$ is an unknown analytic function. We introduce two real functions $\tau$ and $\theta$ satisfying 
$\xi=\mathrm{e}^{\tau-\mathrm{i} \theta}$ and expand $\tau-\mathrm{i} \theta$ as

$$
\tau-\mathrm{i} \theta=\sum_{n=0}^{\infty} a_{n} t^{2 n}=\sum_{n=0}^{\infty} a_{n} \cos 2 n \sigma-\mathrm{i} \sum_{n=1}^{\infty} a_{n} \sin 2 n \sigma,
$$

where the coefficients $a_{n}$ are real. At the interface, $t=\mathrm{e}^{\mathrm{i} \sigma}$ and $\sigma \in[0, \pi]$. Upon noting the identity $x_{\phi}+\mathrm{i} y_{\phi}=1 / \zeta$, it is easy to verify that

$$
\begin{aligned}
& y_{\phi}=\mathrm{e}^{-\tau}(2 \sin \sigma)^{-2+\frac{2 \mu}{\pi}} \sin \left[\theta-\left(3-\frac{\gamma}{\pi}-\frac{2 \mu}{\pi}\right)\left(\sigma-\frac{\pi}{2}\right)\right], \\
& x_{\phi}=\mathrm{e}^{-\tau}(2 \sin \sigma)^{-2+\frac{2 \mu}{\pi}} \cos \left[\theta-\left(3-\frac{\gamma}{\pi}-\frac{2 \mu}{\pi}\right)\left(\sigma-\frac{\pi}{2}\right)\right] .
\end{aligned}
$$

Thus Bernoulli's equation becomes

$$
\frac{R}{2} \mathrm{e}^{2 \tau}(2 \sin \sigma)^{4-\frac{4 \mu}{\pi}}+(R-1) \int_{0}^{\sigma} y_{\phi} \sin \alpha \mathrm{d} \alpha=0 .
$$

To solve equation (4.9), the infinite series in (4.6) are truncated at $n=N-1$ and $N$ collocation points are uniformly distributed on the interval $\left[0, \frac{\pi}{2}\right]$, namely

$$
\sigma_{i}=\frac{\pi(i-1)}{2(N-1)}, \quad i=1,2, \cdots, N
$$

Equation (4.9) is then satisfied at the mesh points $\sigma_{2}, \sigma_{3}, \cdots, \sigma_{N}$ with an additional equation

$$
\int_{0}^{\frac{\pi}{2}} x_{\phi} \sin \sigma \mathrm{d} \sigma=0
$$

which simply means the interface is closed. Finally, this system of $N$ nonlinear equations with $N$ unknowns $\left(a_{0}, a_{1}, \cdots, a_{N-1}\right)$ is solved via Newton's method for a given value of $\gamma$, and $N \geqslant 300$ in all computations. This method of series truncation has been applied successfully to solve many free surface problems (see Vanden-Broeck (2010) for details and references).

Case I. $\gamma=2 \pi / 3$

Numerical results for $\gamma=2 \pi / 3$ (i.e. $\mu=\pi / 6$ ) are shown in Figure 6. A typical profile and corresponding streamlines are plotted in (a) for $R=0.1$. From Bernoulli's equation

$$
R\left(u_{2} u_{2 \sigma}+v_{2} v_{2 \sigma}\right)+(R-1) \sin \sigma \frac{v_{2}}{u_{2}^{2}+v_{2}^{2}}=0,
$$

which is derived from equation (4.1) by taking the derivative with respect to $\sigma$, one can eliminate $R$ by introducing

$$
u_{2}^{\prime}=\sqrt[3]{R /(1-R)} u_{2}, \quad v_{2}^{\prime}=\sqrt[3]{R /(1-R)} v_{2} .
$$

This fact immediately suggests that profiles for different values of $R$ are geometrically similar, which is reasonable since no natural length scale appears in the reduced model. To verify this assertion, numerical solutions are plotted in Figure 6(b) where the profiles from large to small correspond to $R=0.9,0.8,0.6,0.3,0.1$ respectively.

Figure 7 shows comparisons between solutions of the simplified model and the almost self-intersecting solutions obtained from the boundary integral equation method. The black line represents the assumed $120^{\circ}$ angle. To plot these solutions under the same scaling, we enlarge the profiles of the simplified model and then move the profiles vertically so that 

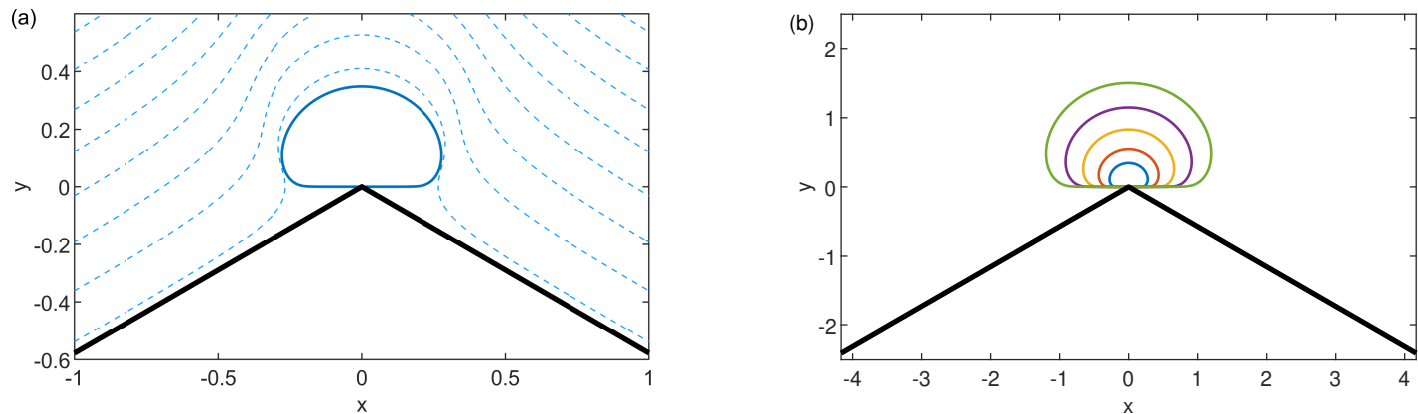

Figure 6: (a) Numerical solution of the simplified model for $\gamma=2 \pi / 3$ and $\mu=\pi / 6$ (solid curve), together with streamlines (dashed curves). (b) Similarity solutions for $R=0.9$, $0.8,0.6,0.3,0.1$ from large to small.
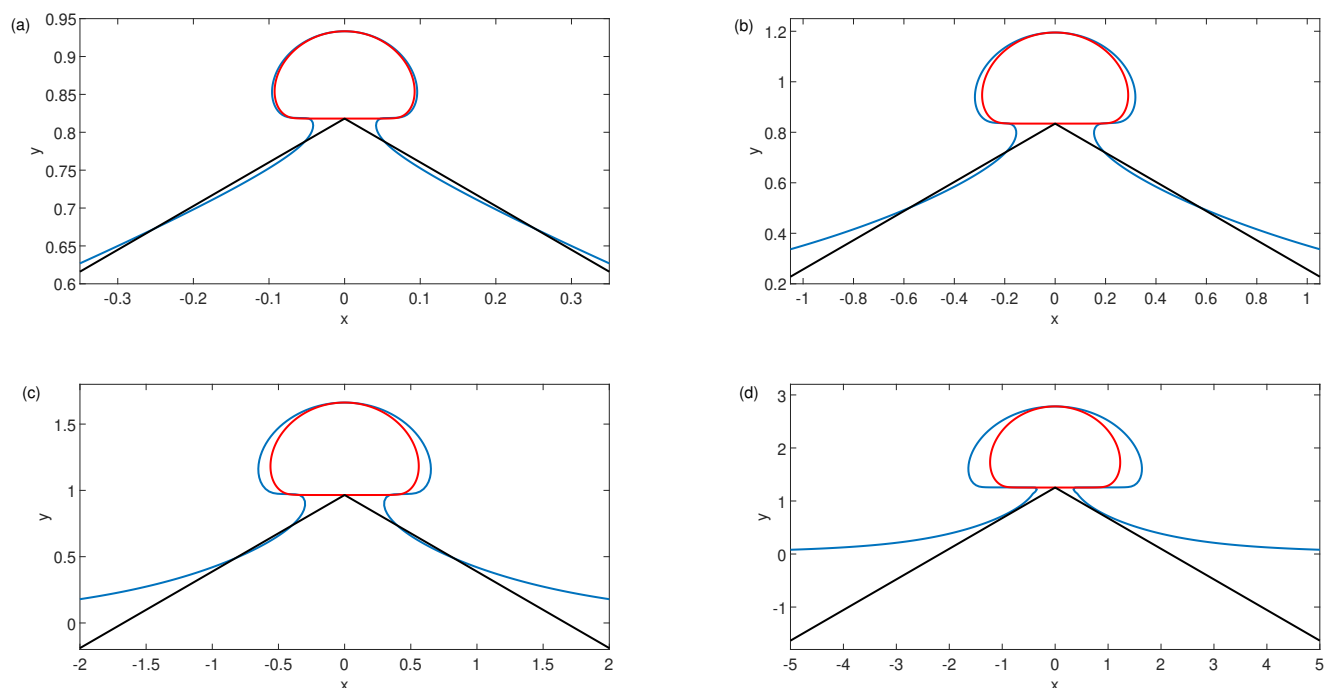

Figure 7: Comparisons between the almost self-intersecting solutions (blue curves) and profiles resulting from the simplified model (red curves). The black lines represent solid walls intersecting at a $120^{\circ}$ angle. (a) $R=0.01$, (b) $R=0.05$, (c) $R=0.1$, (d) $R=0.2$.

their top and bottom match the highest point and flat bottom of the bubble structure of the primitive problem. The density ratios from (a) to (d) are $0.01,0.05,0.1,0.2$ respectively. It is observed that for a small density ratio, the simplified model provides a good approximation to the almost self-intersecting solution of the primitive equations and further to the limiting configuration shown in Figure 1, if it exists.

\section{Case II. $\gamma \neq 2 \pi / 3$}

It is natural to ask what happens to the reduced model when $\gamma \neq \frac{2 \pi}{3}$. In fact, numerical solutions can be found for arbitrary $\gamma \in[0, \pi]$. Four typical solutions with $R=0.1$ are shown in Figure 8.

Two limiting cases, $\gamma=0$ and $\gamma=\pi$, merit special attention. As can be seen from Figure 8 , the profile becomes more and more circular as the value of $\gamma$ is decreased. Therefore, one may expect a perfect circular interface to appear when $\gamma=0$. In fact, it is not difficult to check that $\zeta=\mathrm{i} t\left(1-t^{2}\right) a_{0}$ is an explicit solution of equation (4.12), where $a_{0}=\sqrt[3]{(1-R) / 4 R}$. 

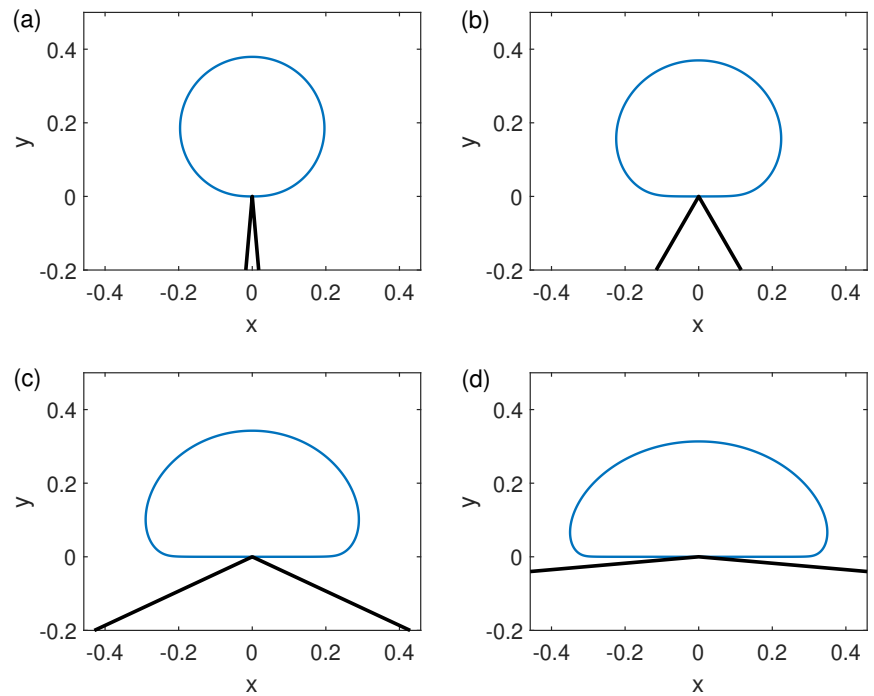

Figure 8: Solutions of the simplified model for (a) $\gamma=\frac{\pi}{18}$, (b) $\gamma=\frac{\pi}{3}$, (c) $\gamma=\frac{13 \pi}{18}$, (d)

$$
\gamma=\frac{17 \pi}{18} \text {. }
$$
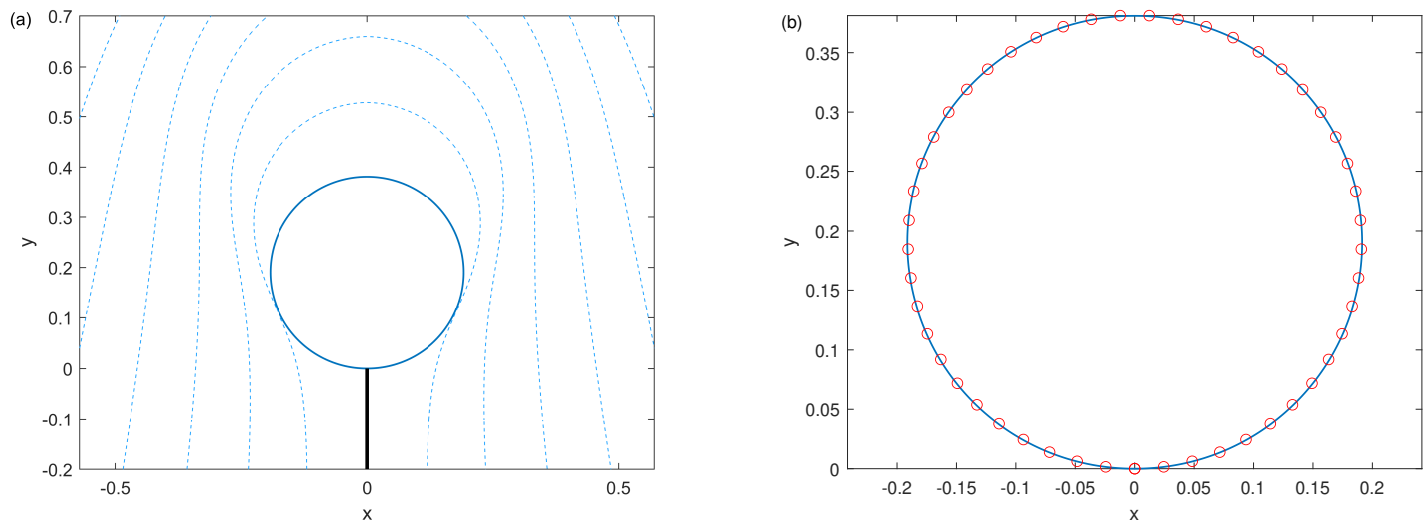

Figure 9: (a) Numerical solution for $\gamma=0$ and $\mu=\frac{\pi}{2}$ (solid curve) and streamlines (dashed curves). (b) Comparison between the numerical solution (solid curve) and theoretical prediction (red circles).

One can then obtain the parametric form of the interface as

$$
x=-\frac{1}{4 a_{0}} \sin 2 \sigma, \quad y=-\frac{1}{4 a_{0}}(\cos 2 \sigma-1),
$$

which is a circle with radius $\frac{1}{4 a_{0}}$. The numerical solution for $R=0.1$ is plotted in figure 9, where the profile and streamlines are displayed in (a) while the comparison with the exact solution is in (b). It thus demonstrates the validity of the numerical algorithm.

For the case of $\gamma=\pi$, the bottom of the fluid bubble entirely attaches to the solid wall, therefore the interface should intersect the solid wall with a $120^{\circ}$ angle and form a stagnation point according to the local analysis. A typical solution for $R=0.1$ is shown in Figure 10 by setting $\mu=\frac{2 \pi}{3}$ and dropping equation (4.11) since the profile is no longer closed at the origin. This type of solution, which describes a still water bubble lying on the flat bottom, exists for all $R \in(0,1)$ due to the geometrical similarity (4.13). Unlike those shown in Figure 6 that represent the limiting solutions for $R \ll 1$, the profile shown in Figure 10 corresponds to 


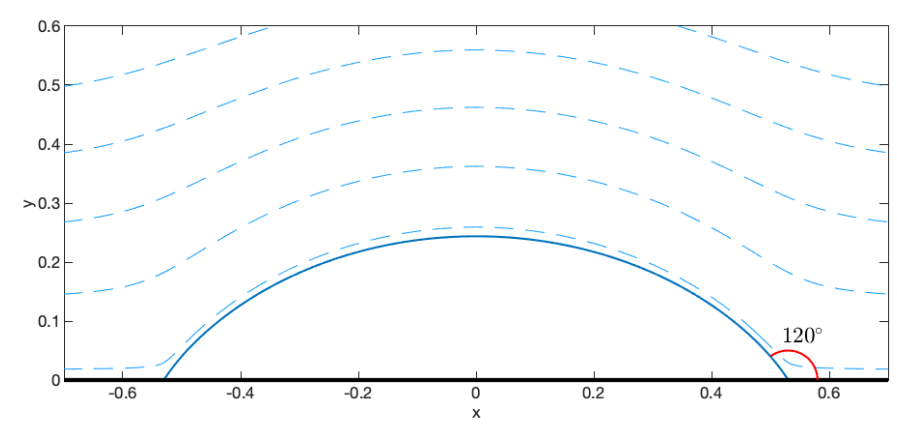

Figure 10: Numerical solution for $\gamma=\pi$ and $\mu=\frac{2 \pi}{3}$ (solid curve), together with streamlines (dashed curves).

another possible limiting configuration of interfacial solitary waves, which appears under the Boussinesq limit, i.e. $R \rightarrow 1$. Such solutions were found by Pullin \& Grimshaw (1988) when the upper fluid is infinitely deep. They proposed that in such a scenario solitary waves are unbounded and calculated the limiting configuration by fixing the wave height and gradually decreasing the lower layer thickness to zero. In particular, they concluded that the limiting interface features a half-lens shape with an approximate aspect ratio (i.e. the ratio of width to height) of 4.36 , which perfectly agrees with 4.353 resulting from our simplified model.

\section{Concluding Remarks}

In conclusion, we have found numerical evidence for a possible limiting configuration of interfacial solitary waves. Overhanging solutions which become almost self-intersecting have been calculated via a boundary integral equation method for various density ratios, strongly suggesting a limiting configuration characterised by a stagnation point at a $120^{\circ}$ angle and a closed fluid bubble on top of the angle (see Figure 1). A simplified model based on these numerical results has been proposed to study the local structure of these singular solutions. Using a series truncation method, we have found exotic solutions depending on the value of $\gamma$, i.e. the angle formed by two intersecting walls. When $\gamma=2 \pi / 3$, the simplified model provides a good approximation to those almost self-intersecting solutions for small density ratios. Solutions for other values of $\gamma$ have also been computed. In particular, we have found an explicit solution featuring a circular profile for $\gamma=0$, and a solution corresponding to another limiting configuration of interfacial solitary waves for $\gamma=\pi$. Furthermore, it is important to mention that the reduced model can also be applied to periodic interfacial waves due to its local nature. Finally, considering the crest instability of the Stokes highest waves (see detailed numerical investigations by Longuet-Higgins \& Tanaka 1997), the Kelvin-Helmholtz instability of interfacial gravity waves (Benjamin \& Bridges 1997), and the Rayleigh-Taylor instability due to the mushroom structure, it is very likely that the almost limiting configurations of progressive interfacial waves are unstable. Therefore, the competition mechanism among different instabilities and the time-evolution of the instability are of particular interest which merit further thorough studies. The only paper we know that provides stability results for interfacial solitary waves is the paper of Kataoka (2006). For small amplitude solitary waves, linear stability analyses based on the Korteweg-de Vries $(\mathrm{KdV})$ equation and its modified version ( $\mathrm{mKdV}$ equation) show that these waves are stable. Using an asymptotic analysis, Kataoka (2006) constructed a general criterion for the stability of interfacial solitary waves with respect to disturbances that are stationary relative to the basic wave. Interesting results were obtained for small density ratios. In particular, Table 1 of Kataoka (2006) provides critical wave amplitudes $H$ at which an exchange of stability first 
occurs for air-water solitary waves $(R=0.0013)$ with various depth ratios $h$. According to this table, all the waves considered in the present paper are unstable. However, the mechanism of the instability is of great interest, since it is related to the theory of wave breaking. As said above, it was suggested that the instability of solitary waves is caused by the crest instability. Assuming that the local crest instability is also the correct mechanism of interfacial solitary wave instability, there is still one important question. Kataoka (2006) found that the exchange of stability occurs at the extremum in the total wave energy. What is the physical connection between the crest instability, which is a local phenomenon, and the extremum in the total wave energy, which is a global quantity? On an apparently completely different problem related to super free fall, Villermaux \& Pomeau (2010) commented on the formation of a concentrated 'nipple' on top of an essentially flat base solution and wondered about the relevance with wave breaking. They noted that wave breaking does occur with standing waves (Taylor 1953) and in nature. The formation of 'nipples' can easily be observed on wave crests. These nipples then bend and splash on the sea surface, forming foam and spume. Is the present study definitely irrelevant to that common but yet unexplained phenomenon? We believe that some interesting dynamics due to the instability of interfacial solitary waves at small density ratios is likely to occur.

\section{Acknowledgements}

This work was supported by the National Natural Science Foundation of China under grant 11772341, by the Strategic Priority Research Program of the Chinese Academy of Sciences under grant XDB22040203, by the European Research Council under the research project ERC-2018-AdG 833125 HIGHWAVE, and in part by EPSRC under grant EP/N018559/1. X.G. would also like to acknowledge the support from the Chinese Scholarship Council.

\section{Declaration of Interests}

The authors report no conflict of interest.

\section{REFERENCES}

Amick, C. J., Fraenkel, L. E. \& Toland, J. F. 1982 On the stokes conjecture for the wave of extreme form. Acta Math. 148(1), 193-214.

Amick, C. J. \& Turner, R. E. L. 1986 A global theory of internal solitary waves in two-fluid systems. Trans. Amer. Math. Soc. 298(2), 431-484.

Benjamin, T. B. \& Bridges, T. J. 1997 Reappraisal of the Kelvin-Helmholtz problem. Part 2. Interaction of the Kelvin-Helmholtz, superharmonic and Benjamin-Feir instabilities. J. Fluid Mech. 333, 327-373.

Daboussy, D., Dias, F. \& Vanden-Broeck, J.-M. 1998 Gravity flows with a free surface of finite extent. Eur. J. Mech. B/Fluids 17, 19-31.

Dias, F. \& Bridges, T. J. 2006 The numerical computation of freely propagating time-dependent irrotational water waves. Fluid Dyn. Res. 38, 803-830.

Funakoshi, M. \& Oikawa, M. 1986 Long internal waves of large amplitude in a two-layer fluid. J. Phys. Soc. Japan 55, 128-144.

Grimshaw, R. H. J. \& Pullin, D. I. 1986 Extreme interfacial waves. Phys. Fluids 29(9), 2802-2807.

Havelock, T. H. 1918 Periodic irrotational waves of finite height. Proc. R. Soc. A 665, 38-51.

Hunter, J. K. \& VAnden-Broeck, J.-M. 1983 Accurate computations for steep solitary waves. J. Fluid Mech. 136, 62-71.

Kataoka, T. 2006 The stability of finite-amplitude interfacial solitary waves. Fluid Dyn. Res. 38, 831-867.

LenAu, C. W. 1966 The solitary wave of maximum amplitude. J. Fluid Mech. 26, 309-320.

Longuet-Higgins, M. S. 1973 On the form of the highest progressive and standing waves in deep water. Proc. R. Soc. A 331, 445-456. 
Longuet-Higgins, M. S. \& Tanaka, M. 1997 On the crest instabilities of steep surface waves. J. Fluid Mech. 336, 51-68.

Maklakov, D. V. 2020 A note on the existence of pure gravity waves at the interface of two fluids. Physica D 401, 132157.

Maklakov, D. V. \& Sharipov, R. R. 2018 Almost limiting configurations of steady interfacial overhanging gravity waves. J. Fluid Mech. 856, 673-708.

Meiron, D. I. \& SAfFman, P. G. 1983 Overhanging interfacial gravity waves of large amplitude. J. Fluid Mech. 129, 213-218.

Окамото, Н., \& Shōı, M. 2001 The Mathematical Theory of Permanent Progressive Water-waves. Advanced Series in Nonlinear Dynamics 20. World Scientific, Singapore.

Pullin, D. I. \& Grimshaw, R. H. J. 1988 Finite-amplitude solitary waves at the interface between two homogeneous fluids. Phys. Fluids 31(12), 3550-3559.

RusÅs, P.-O. \& Grue, J. 2002 Solitary waves and conjugate flows in a three-layer fluid. Eur. J. Mech. B/Fluids 21, 185-206

Schwartz, L. W. 1974 Computer extension and analytic continuation of Stokes' expansion for gravity waves. J. Fluid Mech. 62, 553-578.

SAfFMAn, P. G. \& Yuen, H. C. 1982 Finite-amplitude interfacial waves in the presence of a current. J. Fluid Mech. 123, 459-476.

Sha, H. \& Vanden-Broeck, J.-M. 1993 Two-layer flows past a semicircular obstruction. Phys. Fluids A 5, 2661-2668.

TAylor, G. I. 1953 An experimental study of standing waves. Proc. R. Soc. A 218, 44-59.

Turner, R. E. L. \& VAnden-Broeck, J.-M. 1986 The limiting configuration of interfacial gravity waves. Phys. Fluids 29(2), 372-375.

Turner, R. E. L. \& Vanden-Broeck, J.-M. 1988 Broadening of interfacial solitary waves. Phys. Fluids 31(9), 2486-2490.

VAnden-Broeck, J.-M. 2010 Gravity-Capillary Free-Surface Flows. Cambridge University Press.

VANDEN-Broeck, J.-M. \& Schwartz, L. W. 1979 Numerical computation of steep gravity waves in shallow water. Phys. Fluids 22(10), 1868-1871.

Villermaux, E. \& Pomeau, Y. 2010 Super free fall. J. Fluid Mech. 642, 147-157.

Yamada, H. 1957a Highest waves of permanent type on the surface of deep water. Report Res. Inst. Appl. Mech. Kyushu Univ. 5, 37-52.

Yamada, H. 1957b On the highest solitary wave. Report Res. Inst. Appl. Mech. Kyushu Univ. 5, 53-67. 